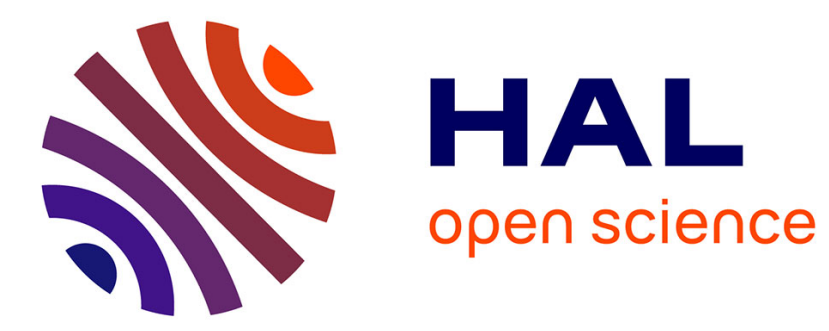

\title{
Benchmarking the BFGS Algorithm on the BBOB-2009 Noisy Testbed
}

Raymond Ros

\section{To cite this version:}

Raymond Ros. Benchmarking the BFGS Algorithm on the BBOB-2009 Noisy Testbed. GECCO, Jul 2009, Montréal, Canada. inria-00377079v2

\section{HAL Id: inria-00377079 \\ https://hal.inria.fr/inria-00377079v2}

Submitted on 22 Oct 2009

HAL is a multi-disciplinary open access archive for the deposit and dissemination of scientific research documents, whether they are published or not. The documents may come from teaching and research institutions in France or abroad, or from public or private research centers.
L'archive ouverte pluridisciplinaire HAL, est destinée au dépôt et à la diffusion de documents scientifiques de niveau recherche, publiés ou non, émanant des établissements d'enseignement et de recherche français ou étrangers, des laboratoires publics ou privés. 


\title{
Benchmarking the BFGS Algorithm on the BBOB-2009 Noisy Testbed
}

\author{
Raymond Ros \\ Univ. Paris-Sud, LRI \\ UMR 8623 / INRIA Saclay, projet TAO \\ F-91405 Orsay, France \\ raymond.ros@Iri.fr
}

\begin{abstract}
The BFGS quasi-Newton method is benchmarked on the noisy BBOB-2009 testbed. A multistart strategy is applied with a maximum number of function evaluations of about $10^{4}$ times the search space dimension.
\end{abstract}

\section{Categories and Subject Descriptors}

G.1.6 [Numerical Analysis]: Optimization-global optimization, unconstrained optimization; F.2.1 [Analysis of Algorithms and Problem Complexity]: Numerical Algorithms and Problems

\section{General Terms}

Algorithms

\section{Keywords}

Benchmarking, Black-box optimization, BFGS, Quasi-newton

\section{INTRODUCTION}

The Broyden-Fletcher-Goldfarb-Shanno (BFGS) method 1. 3, 4, 7] is a real-parameter unconstrained non-linear optimization method. The BFGS method belongs to the class of quasi-Newton methods which, by supposing that the objective function can be locally approximated by a quadratic function near its optimum, tries to approximate the Hessian matrix of this quadratic function. The BFGS method is tested here in its default setting on a testbed of noisy functions.

\section{ALGORITHM PRESENTATION}

Quasi-Newton methods address the problem of unconstrained black-box optimization by the determination of the stationary point of a function using a second-order approximation. The Hessian matrix is iteratively approximated by finding a search direction using the gradient of the current point and operating a line search to find the step size. For

Permission to make digital or hard copies of all or part of this work for personal or classroom use is granted without fee provided that copies are not made or distributed for profit or commercial advantage and that copies bear this notice and the full citation on the first page. To copy otherwise, to republish, to post on servers or to redistribute to lists, requires prior specific permission and/or a fee.

GECCO’09, July 8-12, 2009, Montréal Québec, Canada.

Copyright 2009 ACM 978-1-60558-505-5/09/07 ...\$5.00. each iteration $D+1$ function evaluations (where D denotes the problem dimension) or double if the step size changes, are done to evaluate the gradient and the function value at the current point. A simple stochastic independent restart procedure (as advised in [5]) was added.

\section{EXPERIMENTAL PROCEDURE}

The Matlab implementation of the BFGS method was used. It is accessible using the generic function fminunc (revision 1.1.6.3) that proposes, among others, the BFGS method for the update of the Hessian Matrix. The starting point is chosen uniformly in $[-5,5]^{D}$. The stopping criteria were chosen such that a restart occurs due to numerical errors. The multistart strategy was used with at most 100 restarts to reduce the duration of an experiment. For the same reason, a run is limited to at most $10^{4} \times D$ function evaluations. The entire experiment took roughly 7 hours. The algorithm used is presented in Figure 1. No parameter tuning was done, the CrE 5 is computed to zero.

\section{RESULTS AND DISCUSSION}

Results from experiments according to 5 on the benchmarks functions given in [2, 6] are presented in Figures 2 and 3 and in Tables 1 and 2 . The proposed algorithm only solves $5,3,2,2,2$ and 1 out of 30 functions in dimension $2,3,5,10$, 20 and 40 respectively. The functions solved use the Cauchy noise model only. The Rosenbrock function and Gallagher functions are solved in dimension 2. The sphere with the Cauchy noise is solved for all dimensions tested, though the algorithm scales comparatively worse than on the noiseless sphere. A more severe Cauchy noise model decreases the probability of success of the algorithm. No success was obtained on the multimodal functions with severe noise.

\section{CPU TIMING EXPERIMENT}

For the timing experiment, the proposed algorithm was run on $f_{8}$ and restarted until at least 30 seconds have passed (according to Figure 2 in [5]). The experiments were conducted with an Intel Core 26700 processor $(2.66 \mathrm{GHz})$ with Matlab R2008a on Linux 2.6.24.7. The results were 6.0, 4.7, $3.7,3.0,2.9,2.9$ and $2.8 \times 10^{-4}$ seconds per function evaluations in dimension $2,3,5,10,20,40$ and 80 respectively.

\section{CONCLUSION}

The results of a quasi-Newton method algorithm with restarts were presented. They show the limits of a default Quasi-Newton method on noisy testbeds. 


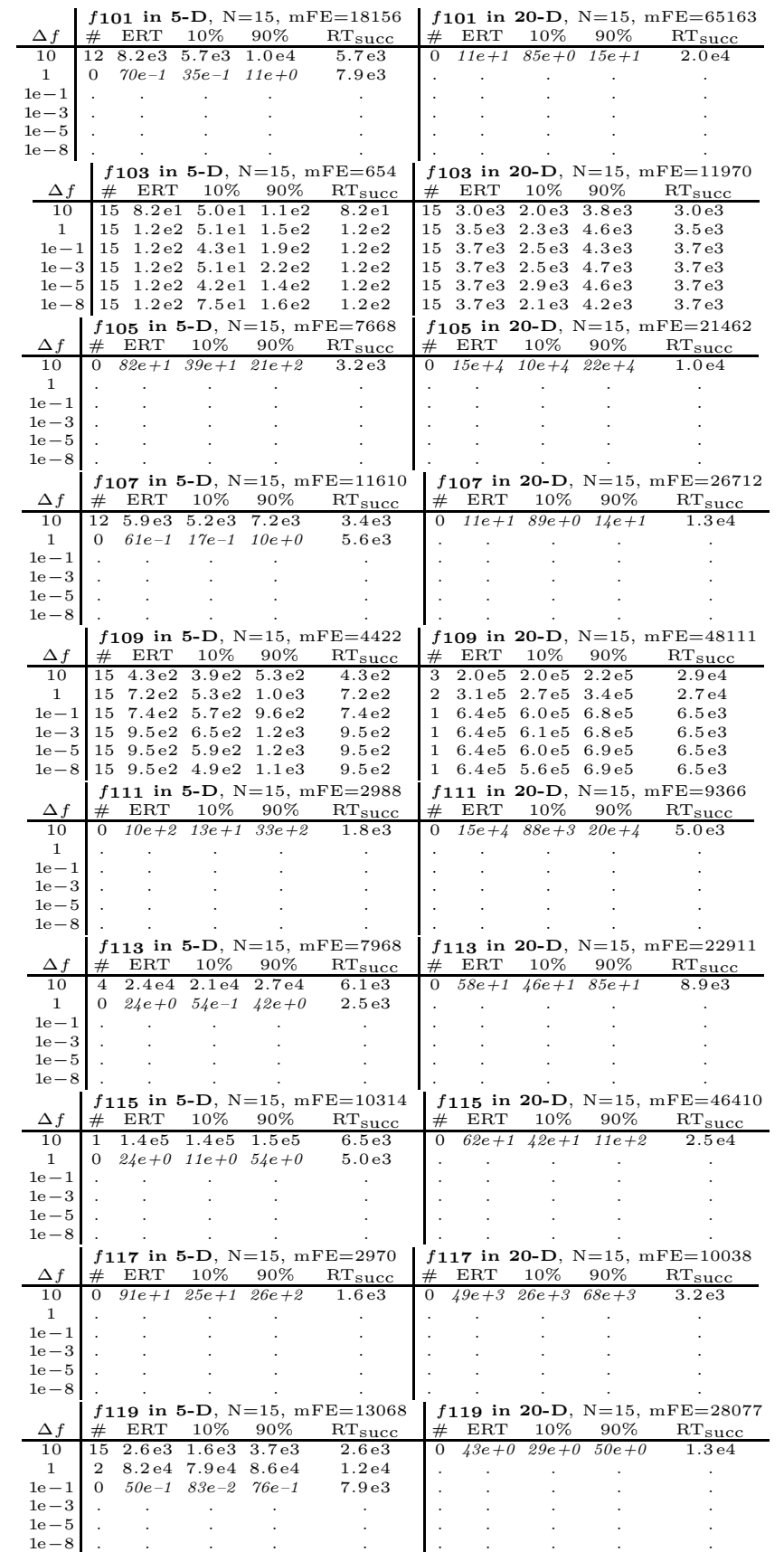

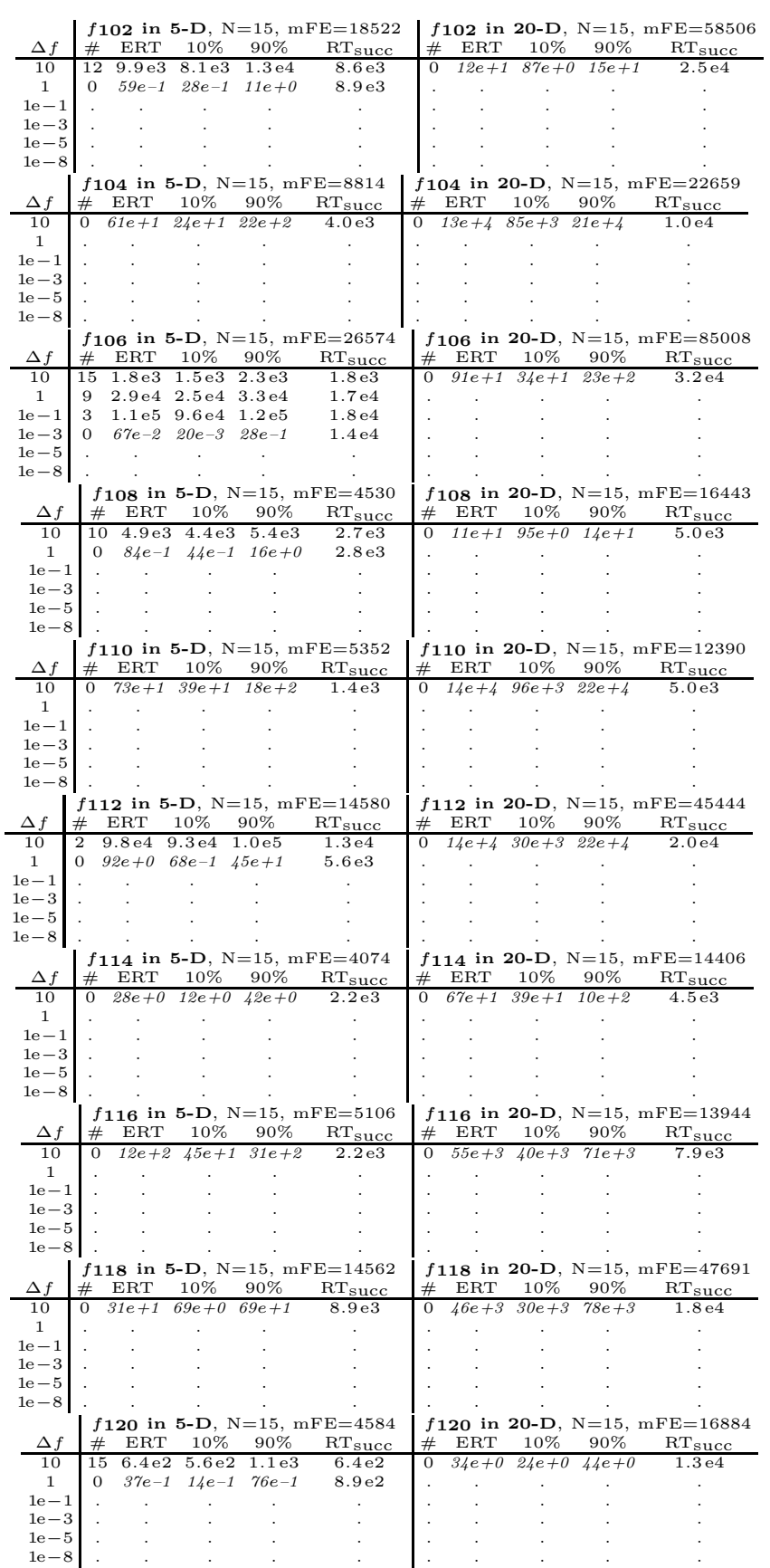

Table 1: Shown are, for functions $f_{101}-f_{120}$ and for a given target difference to the optimal function value $\Delta f$ : the number of successful trials (\#); the expected running time to surpass $f_{\mathrm{opt}}+\Delta f$ (ERT, see Figure 2); the $10 \%$-tile and $90 \%$-tile of the bootstrap distribution of ERT; the average number of function evaluations in successful trials or, if none was successful, as last entry the median number of function evaluations to reach the best function value $\left(\mathbf{R T}_{\text {succ }}\right)$. If $f_{\mathrm{opt}}+\Delta f$ was never reached, figures in italics denote the best achieved $\Delta f$-value of the median trial and the $10 \%$ and $90 \%$-tile trial. Furthermore, $\mathbf{N}$ denotes the number of trials, and $\mathrm{mFE}$ denotes the maximum of number of function evaluations executed in one trial. See Figure 2 for the names of functions. 


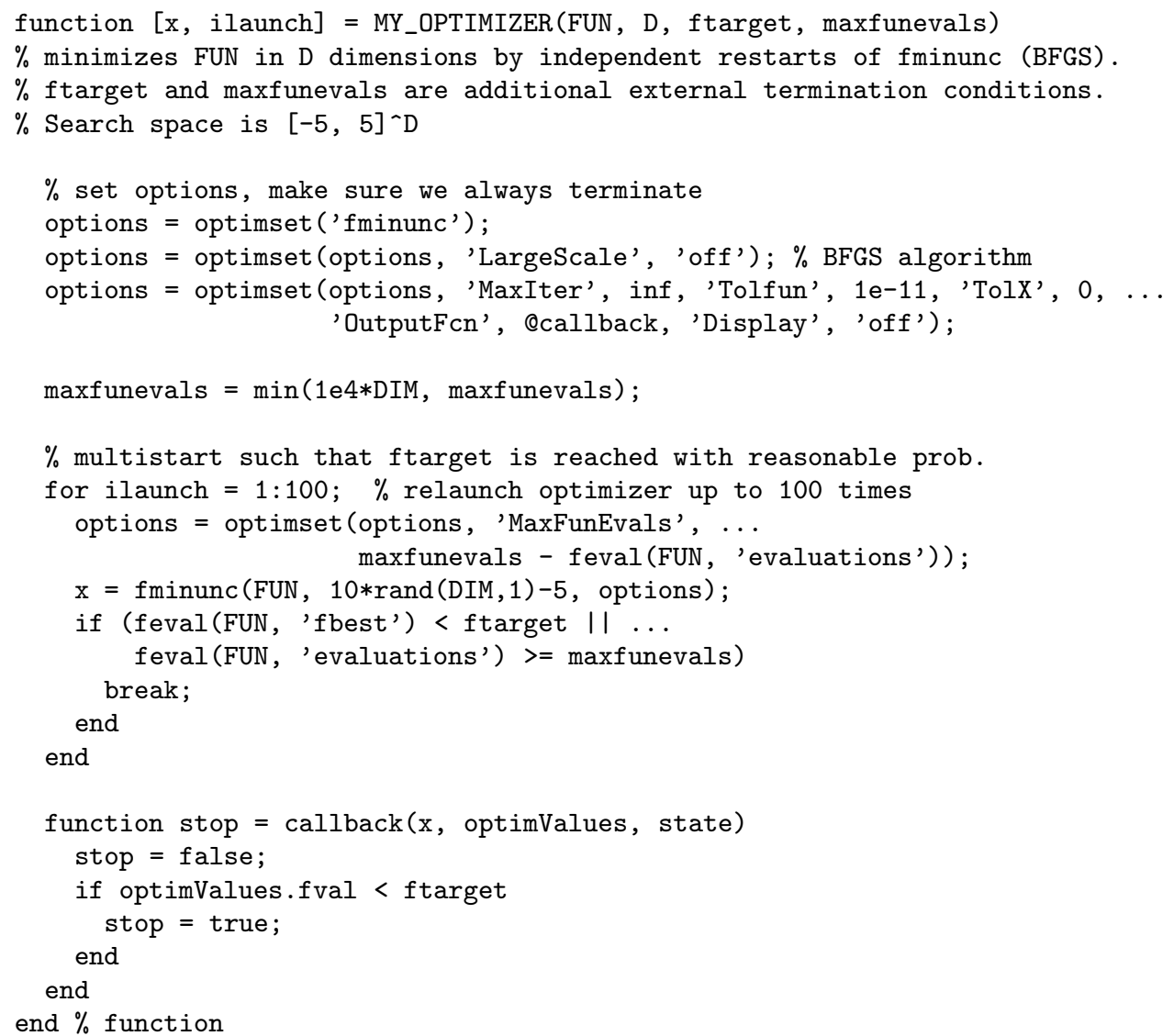

\section{Acknowledgments}

The first author would like to acknowledge the support, help, and work of the BBOB team with particular kudos to Anne Auger, Steffen Finck and Nikolaus Hansen.

\section{REFERENCES}

[1] C. G. Broyden. The convergence of a class of double-rank minimization algorithms. Journal of the Institute of Mathematics and Its Applications, 6:76-90, 1970.

[2] S. Finck, N. Hansen, R. Ros, and A. Auger. Real-parameter black-box optimization benchmarking 2009: Presentation of the noisy functions. Technical Report 2009/21, Research Center PPE, 2009.

[3] R. Fletcher. A new approach to variable metric algorithms. Computer journal, 13:317-322, 1970.

[4] D. Goldfarb. A family of variable metric updates derived by variational means. Mathematics of Computation, 24:23-26, 1970.

[5] N. Hansen, A. Auger, S. Finck, and R. Ros. Real-parameter black-box optimization benchmarking 2009: Experimental setup. Technical Report RR-6828, INRIA, 2009.

[6] N. Hansen, S. Finck, R. Ros, and A. Auger. Real-parameter black-box optimization benchmarking
2009: Noisy functions definitions. Technical Report RR-6869, INRIA, 2009.

[7] D. F. Shanno. Conditioning of quasi-newton methods for function minimization. Mathematics of Computation, 24:647-656, 1970. 

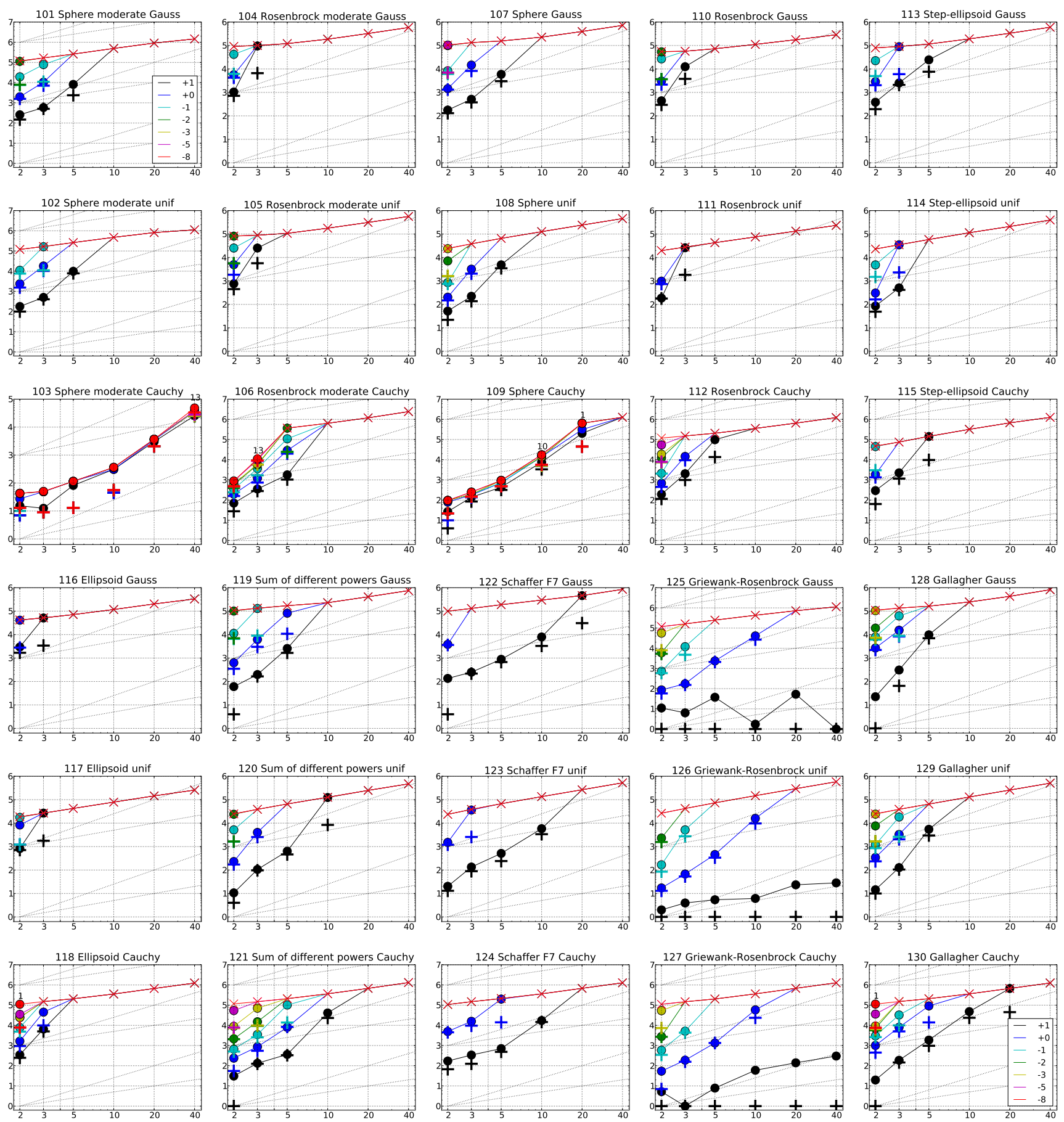

Figure 2: Expected Running Time (ERT, •) to reach $f_{\mathrm{opt}}+\Delta f$ and median number of function evaluations of successful trials $(+)$, shown for $\Delta f=10,1,10^{-1}, 10^{-2}, 10^{-3}, 10^{-5}, 10^{-8}$ (the exponent is given in the legend of $f_{101}$ and $\left.f_{130}\right)$ versus dimension in log-log presentation. The $\operatorname{ERT}(\Delta f)$ equals to \#FEs $(\Delta f)$ divided by the number of successful trials, where a trial is successful if $f_{\mathrm{opt}}+\Delta f$ was surpassed during the trial. The \#FEs $(\Delta f)$ are the total number of function evaluations while $f_{\text {opt }}+\Delta f$ was not surpassed during the trial from all respective trials (successful and unsuccessful), and $f_{\text {opt }}$ denotes the optimal function value. Crosses $(\times)$ indicate the total number of function evaluations $\# \mathrm{FEs}(-\infty)$. Numbers above ERT-symbols indicate the number of successful trials. Annotated numbers on the ordinate are decimal logarithms. Additional grid lines show linear and quadratic scaling. 

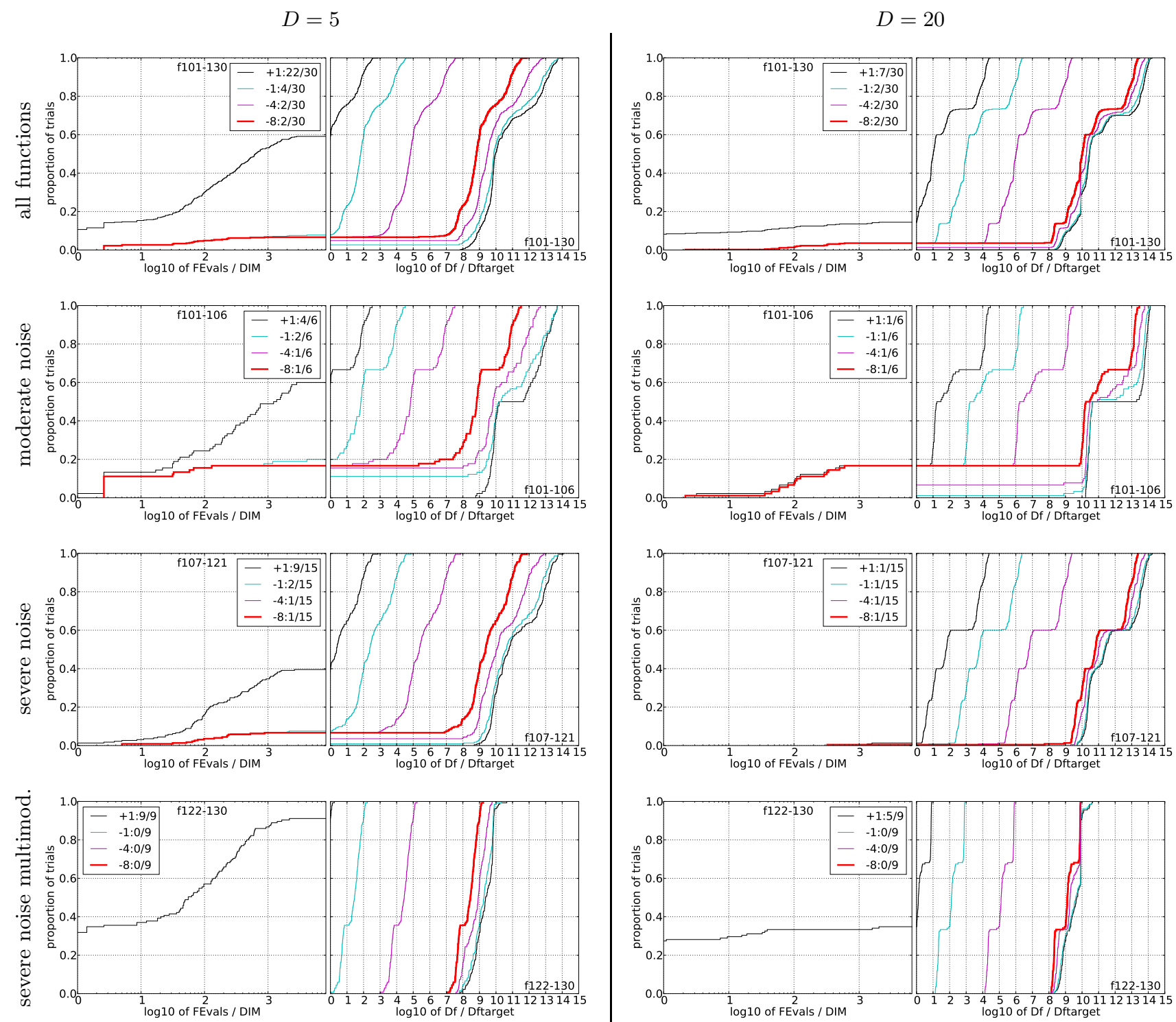

Figure 3: Empirical cumulative distribution functions (ECDFs), plotting the fraction of trials versus running time (left subplots) or versus $\Delta f$ (right subplots). The thick red line represents the best achieved results. Left subplots: ECDF of the running time (number of function evaluations), divided by search space dimension $D$, to fall below $f_{\text {opt }}+\Delta f$ with $\Delta f=10^{k}$, where $k$ is the first value in the legend. Right subplots: ECDF of the best achieved $\Delta f$ divided by $10^{k}$ (upper left lines in continuation of the left subplot), and best achieved $\Delta f$ divided by $10^{-8}$ for running times of $D, 10 D, 100 \mathrm{D} \ldots$ function evaluations (from right to left cycling blackcyan-magenta). Top row: all results from all functions; second row: moderate noise functions; third row: severe noise functions; fourth row: severe noise and highly-multimodal functions. The legends indicate the number of functions that were solved in at least one trial. FEvals denotes number of function evaluations, $D$ and DIM denote search space dimension, and $\Delta f$ and Df denote the difference to the optimal function value. 

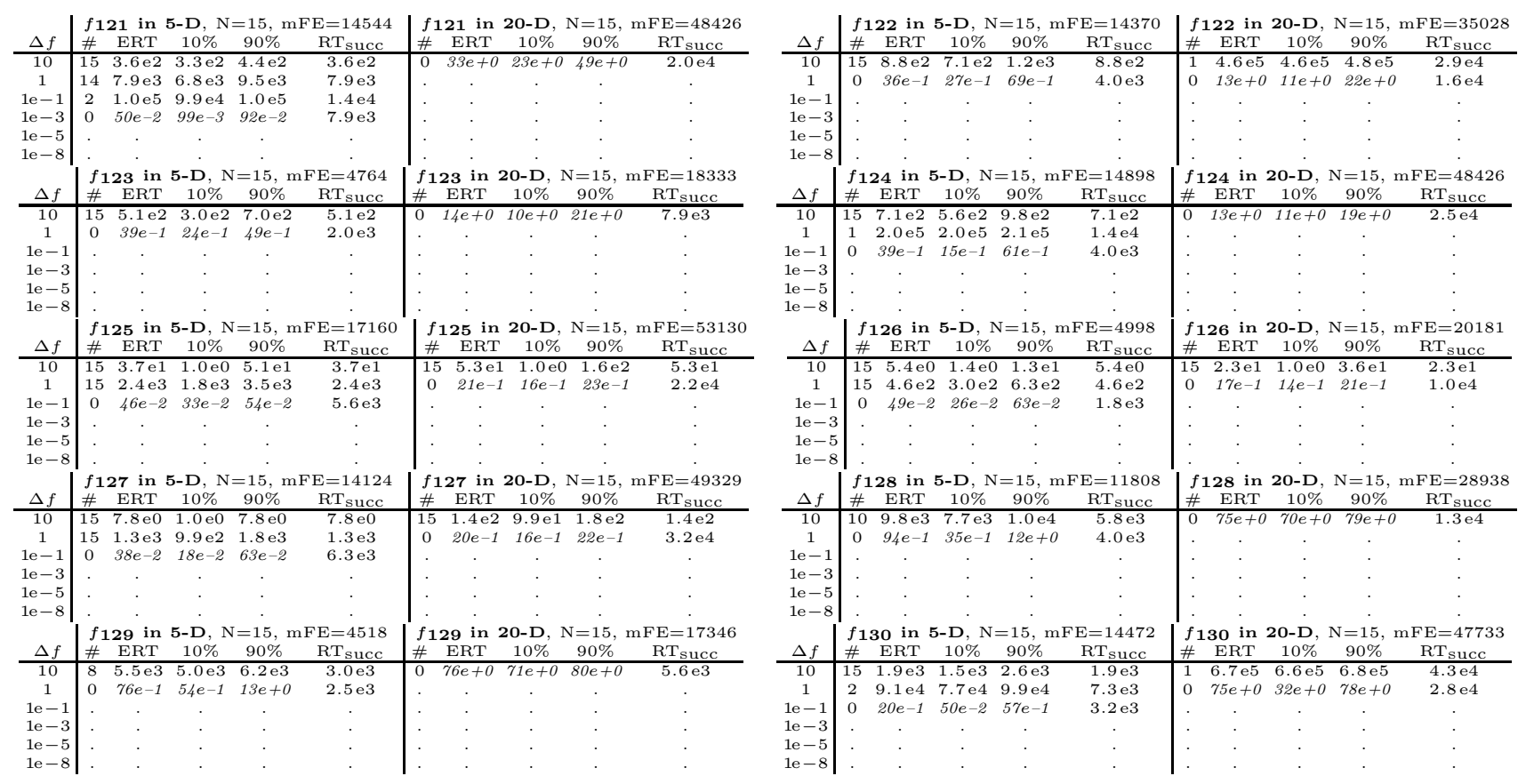

Table 2: Shown are, for functions $f_{121}-f_{130}$ and for a given target difference to the optimal function value $\Delta f$ : the number of successful trials (\#); the expected running time to surpass $f_{\mathrm{opt}}+\Delta f$ (ERT, see Figure 2); the 10\%-tile and $90 \%$-tile of the bootstrap distribution of ERT; the average number of function evaluations in successful trials or, if none was successful, as last entry the median number of function evaluations to reach the best function value $\left(\mathbf{R T}_{\text {succ }}\right)$. If $f_{\mathrm{opt}}+\Delta f$ was never reached, figures in italics denote the best achieved $\Delta f$-value of the median trial and the $10 \%$ and $90 \%$-tile trial. Furthermore, $\mathbf{N}$ denotes the number of trials, and $\mathrm{mFE}$ denotes the maximum of number of function evaluations executed in one trial. See Figure 2 for the names of functions. 